\title{
Cellular mechanism of eccentric-induced muscle injury and its relationship with sarcomere heterogeneity
}

\author{
Seung Jun Choi* \\ Division of Sports and Health Science, Kyungsung University, Busan, Korea
}

Activity-induced muscle injury and dysfunction have been identified as key components of musculoskeletal injuries. These injuries often occur following eccentric contractions, when the muscle is under tension and stretched by a force that is greater than the force generated by the muscle. Many daily activities require muscles to perform eccentric contractions, including walking (or running) downhill or down stairs, lowering heavy objects, and landing from a jump. Injuries often occur when these activities are performed at high intensity or for prolonged periods of time. General features of eccentric-induced muscle injury are well documented and include disruption of intracellular muscle structure, prolonged muscle weakness and dysfunction, a delayed-onset muscle soreness, and inflammation. Several weeks are required for

\section{INTRODUCTION}

Musculoskeletal disorders and injuries are one of the common health problems. The key components of musculoskeletal injuries have been identified as an activity-induced muscle injury. Skeletal muscle injury typically occurs following excessive muscular activity, particularly if the muscle is under tension and stretched by an opposing force that is greater than the force generated by the muscle. Under these conditions, muscle can function as brakes to slow limb movement, or shock absorbers to dissipate energy. A braking or shock absorbing contraction is called an eccentric, plyometric, or lengthening contraction. Many daily activities require muscle to perform eccentric contraction, including walking (or running) downhill or down stairs, lowering heavy objects, and landing from a jump etc.

Typical functional consequences of eccentric contractions are a reduction in peak power, a loss in peak force, a slowing of shorten- the affected tissue to fully regenerate and recover from eccentric-induced muscle injury. Possible mechanisms responsible for eccentric-induced muscle injury are activation impairment and structural disruption of the sarcomere. These two factors seem to be the main sources of eccentric-induced muscle injury. Rather than being separate mechanisms they may be complimentary and interact with each other. Therefore, in this review we will focus on the two main cellular mechanism of muscle cell injury following accustomed eccentric contraction.

Keywords: Eccentric contraction, Cross-bridge, Excitation-contraction coupling, Sarcomere heterogeneity ing velocity, and a shift in optimal muscle length to a longer length (Brockett et al., 2001; Jones et al., 1997; Morgan and Proske, 2004). Thus, it resembles muscle fatigue in several characteristics, such as a decline in force, shortening velocity, and power. The difference between muscle fatigue and damage is whether muscle function is able to recover within a short period of time. In other words, muscle fatigue is a generally showed short-lived dysfunction, whereas muscle damage is a prolonged dysfunction that needs several days for complete recovery (Hough, 1900).

Because of prevalence and costs of muscle injury, it is important to understand the mechanisms underlying the pathology of muscle injuries. This knowledge may then lead to effective ways of preventing, treating, and rehabilitating injuries. Armstrong et al. (1991) proposed that contraction-induced injury was due to an initiating event that targeted a population of susceptible cells. The initiating event in eccentric-induced muscle injury is most likely mechanical in nature (Friden and Lieber, 2001). Morgan and Proske
${ }^{*}$ Corresponding author: Seung Jun Choi

Division of Sports and Health Science, Kyungsung University, 309 Suyoung-ro,

Nam-gu, Busan 608-736, Korea

Tel: +82-51-663-4955, Fax: +82-51-663-4956, E-mail: choisj@ks.ac.kr

Received: July 28, 2014 / Accepted: August 9, 2014
This is an Open Access article distributed under the terms of the Creative Commons Attribution Non-Commercial License (http://creativecommons.org/licenses/by-nc/3.0/) which permits unrestricted non-commercial use, distribution, and reproduction in any medium, provided the original work is properly cited. 
(2004) propose that sarcomere length heterogeneity leads to a few weaker sarcomeres when muscle is stretched or lengthened. These weak sarcomeres stretch more rapidly and widely than other stronger sarcomeres. These overstretched sarcomeres lead to structural distortions that are propagated longitudinally and rapidly through the myofibril, leading to physical disruption of the sarcomere, membrane damage, and impairment of excitation contraction coupling (Allen, 2001; Lieber and Friden, 2002; Proske and Allen, 2005). Muscle stretch may open stretch activated $\mathrm{Ca}^{2+}$ channels and $\mathrm{Ca}^{2+}$ may enter into the cell via these channels or through ruptures in the sarcolemma (Overgaard et al., 2002). All of this leads to an increase in intracelluar $\mathrm{Ca}^{2+}$ levels (Balnave and Allen, 1995; Ingalls et al., 1998), which may activate intracellular proteases, such as calpain (Belcastro, 1993). Leukocyte infiltration (neutrophils, natural killer cells, marcrophage) and increased pro-inflammatory cytokines (interleukin- $1 \beta$, interleukin- 6 , tumor necrosis factor- $\alpha$ ) are attracted to the injured site and contribute to further degradation of muscle tissue (Peake et al., 2005). Activation of myogenic precursor cell, or satellite cells, to proliferate and differentiate is required for regeneration of new myofibers and reconstitution of contractile structure (Charge and Rudnicki, 2004). Therefore, the aim of this review is to focus attention particularly on the two main cellular mechanism of muscle cell injury following accustomed eccentric contraction.

\section{EXCITATION AND CONTRACTION (EC) COUPLING IMPAIRMENT}

The first possible mechanism is a failure of $\mathrm{Ca}^{2+}$ release and uptake. Warren and associates proposed that impaired EC coupling plays a major role in eccentric-induced muscle injury (Ingalls et al., 1998; Warren et al., 1993).

The failure of EC coupling has been demonstrated after eccentric exercise (Balnave and Allen, 1995), and it suggests that less calcium is released per action potential. Caffeine bypasses the normal EC coupling process and directly opens the RyR, resulting in release of $\mathrm{Ca}^{2+}$ from SR (Endo, 1977; Martonosi, 1984). Warren hypothesized that if reduced force, induced by eccentric contraction, is able to be bypassed by caffeine, then the reduced force can be attributed to EC coupling failure. Under this hypothesis, he studied that the mouse soleus muscle were exposed to bicarbonate buffer containing caffeine after 20 eccentric contractions (Warren et al., 1993). The caffeine-elicited isometric force in injured mouse muscles was not different from the control muscle, even though maximal isometric tetanic force of injured muscle was reduced
43\%. Accordingly, Warren and colleagues proposed an impaired EC coupling system as a main source of dysfunction.

By using a skinned single fiber preparation, the EC coupling pathway can be bypassed and the fiber activated by directly application of saturating $\mathrm{Ca}^{2+}$. Thus, under this condition, the reduced maximal $\mathrm{Ca}^{2+}$-activated force can be attributed to the disruption or alteration of the force-generating and transmitting structures. Thus, Warren et al. (1993) tested the force reduction in the isolated EDL muscle after 15 eccentric contractions, and also measured the maximal $\mathrm{Ca}^{2+}$-activated force on skinned muscle fiber from that isolated EDL muscle. The result showed that the maximal isometric force of the isolated muscle was reduced by $69 \%$, whereas the maximal $\mathrm{Ca}^{2+}$-activated force was reduced by $34 \%$. Therefore, they concluded that the physical disruption of muscle ultrastructure may explain about half of the strength loss of the muscle.

Balnave and Allen (1995) directly measured $\mathrm{Ca}^{2+}$ release. They conclude that $\mathrm{Ca}^{2+}$-activated force was not significantly affected after 10 eccentric contractions at $25 \%$ strain on living muscle fibers. However, with $50 \%$ strain, the about $79 \%$ of the force reduction can be attributed to the disruption of the sarcomere.

To investigate how long the $\mathrm{EC}$ coupling failure contributes to the force deficit, measurements were made at $0,1,3,5$, and 14 days after in vivo 150 eccentric contractions. The study concluded that during the first 5 days after injury, EC coupling failure is thought to account for about $57-75 \%$ of the strength deficit. The EC coupling failure is diminishing by 5 days after and is resolved by 14 days after the injury (Ingalls et al., 1998). Thus, the disrupted muscle ultrastructure is thought to account for about $25-43 \%$ of the strength deficit.

The possible failure sites in the EC coupling pathway are, the sarcolemma, the t-tubule, the DHPR, the linkage between the DHPR and the RyR, the RyR, and the SR. First, at the sarcolemma, EMG amplitude was reduced 9\% after eccentric contractions (Warren et al., 1999), suggesting a failure in action potential propagation. However, because force was reduced $47 \%$, action potential failure is not considered a major contributor to dysfunction. Second, the ability of the t-tubule to conduct action potential and the intrinsic properties of the DHPR do not appear to be impaired significantly. Because the potassium $\left(\mathrm{K}^{+}\right)$induced force was reduced proportionally with maximal isometric force after 150 in vivo eccentric contractions, and it suggests the failure site of EC coupling process is below the level of the DHPR (Ingalls et al., 1998) since $\mathrm{K}^{+}$trigger muscle contraction by depolarization of the t-tubule and voltage sensor (Dulhunty, 1991). More recently the t-tubules have been observed to be broken after $40 \%$ of strain, 
and it may due to movement, originating from the heterogeneity of sarcomere length (Yeung et al., 2002). Third, the linkage between the DHPR and the RyR is thought to be most fragile site. Warren et al. (1999) proposed that the EC coupling failure result from the weak mechanical link between DHPR and RyR, because the conformational change of DHPR may be failed to be communicated to the RyR. Lastly, at the SR, the intrinsic function of ryanodine receptor decreases by $16 \%$ over the first 3 days after in vivo eccentric injury (Ingalls et al., 2004), but this progressive worsening of SR function is not associated with a further impairment in muscle functions, because the rates of $\mathrm{Ca}^{2+}$ release and uptake by the SR are not significantly impaired (Warren et al., 1993).

\section{CROSS-BRIDGE IMPAIRMENT}

It is hard to conclude that all portion of the muscle dysfunction induced by eccentric contraction is caused by $\mathrm{EC}$ coupling failure only. As mentioned above, Balnave and Allen (1995) founded evidence for both the EC coupling impairment and the structural disruption with $25 \%$ and $50 \%$ of strain, respectively. Also, some find that the force deficit cannot be overcome by caffeine treatment in frog fibers (Morgan et al., 1996), and toad muscle (Allen, 2001). Finally, impaired EC coupling system cannot readily explain two significant characteristic features associated with damage from eccentric contraction: a shift in the length-tension relationship of the muscle and increased passive tension.

An alternative possible mechanism for eccentric-induced muscle dysfunction is based on the heterogeneity of sarcomere length and the length-tension relationship. This is the called "popping sarcomere hypothesis" proposed by Morgan and Proske (2004). It is derived from two characteristics of muscle contraction: the produced force by eccentric contraction is rather greater than the force produced by concentric contraction and the presence of the unstable sarcomere lengthened onto the descending limb of their length tension relationship. The length tension relationship refers to a linear decrease in active tension as the sarcomere length get shorten or lengthen to outside of optimal length, and it is correlated with decreasing overlap of thick and thin filaments (Gordon et al., 1966).

The popping sarcomere hypothesis assumes the presence of irregular sarcomere lengths. This results in eccentric-induced muscle injury because the longest sarcomeres become the weakest sarcomere when stretched out to the descending limb of their lengthtension relationship. Thus, these longer, weaker sarcomeres are stretched more rapidly and widely than other sarcomeres. As a consequence, weaker sarcomeres take up most of the stretch until its total tension is balanced by the tension produced by in series sarcomeres, which are on the plateau of their length tension relationship. The thin and thick filaments making up this overstretched sarcomere may not re-interdigitate when the muscle relaxes (Talbot and Morgan, 1996). If this occurs continually, more and more sarcomeres will become overstretched and dysfunction. Once one or more sarcomere has become disrupted, the damage may expand longitudinally to adjacent sarcomeres in the myofibril and transversely to adjacent myofibrils. The longitudinal and transverse propagation of damage may also impact membranes of the sarcoplasmic reticulum, transverse tubules, and sarcolemma. This could then initiate an uncontrolled movement of $\mathrm{Ca}^{2+}$ into the sarcoplasm (Yeung and Allen, 2004), triggering the next stage in the damage process. According to this hypothesis, the disruption of muscle ultrastructure precedes EC coupling failure, and it is proposed as a main factor leading to reduced functional properties after eccentric contractions (Proske and Allen, 2005).

One consequence of disrupted sarcomeres is a shift in optimal length to longer lengths (Brockett et al., 2001; Jones et al., 1997; Morgan and Proske, 2004). This shift in optimal length is a reliable and useful measure of the amount of eccentric-induced damage (Talbot and Morgan, 1998). It is proposed that this shift in muscle optimal length after the eccentric contractions is due to the disrupted sarcomeres, which lie scattered at random along the myofibril. The presence of these disrupted sarcomeres in parallel with undisrupted sarcomeres increases the series compliance of the fiber, and the increased series compliance leads to a shift in the muscle's optimal length for peak active force toward longer muscle lengths (Morgan et al., 1996).

Another consequence of the disruption of sarcomere is the rise in passive tension immediately after eccentric contraction observed in isolated muscle (Whitehead et al., 2001) and single fibers (Joumaa et al., 2007). It is greatest in the region of the muscle's optimal length (Whitehead et al., 2003). The possible mechanism of this rising passive tension is that uncontrolled $\mathrm{Ca}^{2+}$ released into the sarcoplasm by eccentric contractions may result in the activation the contractile filaments to develop an injury contracture, thus it causes rising passive tension (Whitehead et al., 2003). In addition, the uncontrolled release of $\mathrm{Ca}^{2+}$ into the sarcoplasm may bind with titin, raising the passive tension. The PEVK domain of titin binds $\mathrm{Ca}^{2+}$ with high affinity, resulting in the rising of passive tension of PEVK domain (Labeit et al., 2003), which is the main contributor to passive tension of titin (Horowits, 1999). 


\section{SARCOMERE HETEROGENEITY}

Recently, sarcomere heterogeneity was proposed as a causal relationship between sarcomere strain and fiber bundle injury by eccentric contractions (Patel et al., 2004). This study found that heterogeneity of sarcomeres was increased as the activated muscle bundles underwent successive stretches, and it found a high correlation $\left(r^{2}=0.87\right)$ between sarcomere strain and the relative force deficit. Furthermore, the progressive increase in heterogeneity of sarcomere length is inversely related to maximal eccentric force with a correlation coefficient ranging from 0.61 to 0.93 . Because the weak sarcomeres are beyond their optimal length, and become weaker or longer as heterogeneity increases, this result in a lower maximal eccentric force. This group also concluded that the contribution of EC coupling to eccentric-induced muscle dysfunction is relatively small, especially as strain increases. Accordingly, the high correlation between sarcomere heterogeneity, and force deficit and maximal eccentric force agree with popping sarcomere hypothesis.

\section{CONCLUSIONS}

The initial sequence of events underlying eccentric muscle dysfunction begins with the disruption of a sub-population of sarcomeres due to the heterogeneity of sarcomere length. Then, structural distortions triggered by the disruptions of these sarcomeres leads to membrane damage, which interferes with EC coupling. At the same time, the accompanying stress applied to the sarcolemma leads to opening of cation channels into rupture of the sarcolemma. All of this produces an increase in intracellular $\mathrm{Ca}^{2+}$ levels which triggers proteolysis associated and fiber breakdown. A key point in that the process is initiated at the level of the myofilament lattice of the sarcomere.

\section{CONFLICT OF INTEREST}

No potential conflict of interest relevant to this article was reported.

\section{ACKNOWLEDGMENTS}

This research was supported by Kyungsung University Research Grants in 2012.

\section{REFERENCES}

Allen DG. Eccentric muscle damage: Mechanisms of early reduction of force. Acta Physiol Scand 2001;171:311-319.

Armstrong RB, Warren GL, Warren JA. Mechanisms of exercise-induced muscle fibre injury. Sports Med 1991;12:184-207.

Balnave CD, Allen DG. Intracellular calcium and force in single mouse muscle fibres following repeated contractions with stretch. J Physiol 1995;488:25-36.

Belcastro AN. Skeletal muscle calcium-activated neutral protease (calpain) with exercise. J Appl Physiol 1993;74:1381-1386.

Brockett CL, Morgan DL, Proske U. Human hamstring muscles adapt to eccentric exercise by changing optimum length. Med Sci Sports Exerc 2001;33:783-790.

Charge SB, Rudnicki MA. Cellular and molecular regulation of muscle regeneration. Physiol Rev 2004;84:209-238.

Dulhunty AF. Activation and inactivation of excitation-contraction coupling in rat soleus muscle. J Physiol 1991;439:605-626.

Endo M. Calcium release from the sarcoplasmic reticulum. Physiol Rev 1977;57:71-108.

Friden J, Lieber RL. Serum creatine kinase level is a poor predictor of muscle function after injury. Scand J Med Sci Sports 2001;11:126-127.

Gordon AM, Huxley AF, Julian FJ. The variation in isometric tension with sarcomere length in vertebrate muscle fibres. J Physiol 1966;184:170192.

Horowits R. The physiological role of titin in striated muscle. Rev Physiol Biochem Pharmacol 1999;138:57-96.

Hough T. Ergographic studies in muscular fatigue and soreness. J Boston Soc Med Sci 1900;5:81-92.

Ingalls C, Warren G, Zhang J, Hamilton S, Armstrong R. Dihydropyridine and ryanodine receptor binding after eccentric contractions in mouse skeletal muscle. J Appl Physiol 2004;96:1619-1625.

Ingalls CP, Warren GL, Williams JH, Ward CW, Armstrong RB. E-C coupling failure in mouse EDL muscle after in vivo eccentric contractions. J Appl Physiol 1998;85:58-67.

Jones C, Allen T, Talbot J, Morgan D, Proske U. Changes in the mechanical properties of human and amphibian muscle after eccentric exercise. Eur J Appl Physiol Occup Physiol 1997;76:21-31.

Joumaa V, Rassier D, Leonard T, Herzog W. Passive force enhancement in single myofibrils. Pflugers Archiv European Journal of Physiology 2007;455:367-371.

Labeit D, Watanabe K, Witt C, Fujita H, Wu Y, Lahmers S, Funck T, Labeit S, Granzier H. Calcium-dependent molecular spring elements in the giant protein titin. Proc Natl Acad Sci U S A 2003;100:13716-13721.

Lieber RL, Friden J. Mechanisms of muscle injury gleaned from animal 
models. Am J Phys Med Rehabil 2002;81:S70-79.

Martonosi AN. Mechanisms of $\mathrm{Ca}^{2+}$ release from sarcoplasmic reticulum of skeletal muscle. Physiol Rev 1984;64:1240-1320.

Morgan DL, Claflin DR, Julian FJ. The effects of repeated active stretches on tension generation and myoplasmic calcium in frog single muscle fibres. J Physiol 1996;497(Pt 3):665-674.

Morgan DL, Proske U. Popping sarcomere hypothesis explains stretch-induced muscle damage. Clin Exp Pharmacol Physiol 2004;31:541-545.

Overgaard K, Lindstrom T, Ingemann-Hansen T, Clausen T. Membrane leakage and increased content of $\mathrm{Na}^{+}-\mathrm{K}^{+}$pumps and $\mathrm{Ca}^{2+}$ in human muscle after a 100-km run. J Appl Physiol 2002;92:1891-1898.

Patel TJ, Das R, Friden J, Lutz GJ, Lieber RL. Sarcomere strain and heterogeneity correlate with injury to frog skeletal muscle fiber bundles. J Appl Physiol 2004;97:1803-1813.

Peake J, Nosaka K, Suzuki K. Characterization of inflammatory responses to eccentric exercise in humans. Exerc Immunol Rev 2005;11:64-85.

Proske U, Allen TJ. Damage to skeletal muscle from eccentric exercise. Exerc Sport Sci Rev 2005;33:98-104.

Talbot JA, Morgan DL. Quantitative analysis of sarcomere non-uniformities in active muscle following a stretch. J Muscle Res Cell Motil 1996; 17:261-268.
Talbot JA, Morgan DL. The effects of stretch parameters on eccentric exercise-induced damage to toad skeletal muscle. J Muscle Res Cell Motil 1998;19:237-245.

Warren G, Ingalls C, Shah S, Armstrong R. Uncoupling of in vivo torque production from EMG in mouse muscles injured by eccentric contractions. J Physiol 1999;515(Pt2):609-619.

Warren GL, Lowe DA, Hayes DA, Karwoski CJ, Prior BM, Armstrong RB. Excitation failure in eccentric contraction-induced injury of mouse soleus muscle. J Physiol 1993;468:487-499.

Whitehead N, Morgan D, Gregory J, Proske U. Rises in whole muscle passive tension of mammalian muscle after eccentric contractions at different lengths. J Appl Physiol 2003;95:1224-1234.

Whitehead N, Weerakkody N, Gregory J, Morgan D, Proske U. Changes in passive tension of muscle in humans and animals after eccentric exercise. J Physiol 2001;533:593-604.

Yeung EW, Allen DG: Stretch-activated channels in stretch-induced muscle damage. Role in muscular dystrophy. Clin Exp Pharmacol Physiol 2004;31:551-556.

Yeung EW, Balnave CD, Ballard HJ, Bourreau JP, Allen DG. Development of t-tubular vacuoles in eccentrically damaged mouse muscle fibres. J Physiol 2002;540:581-592. 